\title{
Training of Precise Movements in 3D or 2D for Persons with Parkinson's Disease?
}

Imre Cikajlo, Zlatko Matjacic, Helena Burger, Karmen Peterlin Potisk

University Rehabilitation Institute

Linhartova 51

SI-1000 Ljubljana. Slovakia

imre.cikajlo@ir-rs.si, zlatko.matjacic@ir-rs.si, helena.burger@ir-rs.si,karmen.potisk@ir-rs.si

ABSTRACT: The 10 Cubes for training and assessment of precise movements with upper extremities has been developed for $3 D$ virtual reality. The system comprises of an infrared stereo camera for hand/finger movements detection and a $3 D$ head mounted device.

In the small scale study 13 persons with Parkinson's disease participated. The participants were randomized into 2 groups; one using a laptop and the other using a 3D head mounted device. The 2-week protocol with 10 sessions, each lasting for 30 min revealed that participants improved some functions by clinical means besides the successful game performance. The kinematics of the hand movements and the outcomes of the clinical test Box \& blocks improved for both groups.

Keywords: Parkinson's Disease, 3 D imaging, Virtual Reality, Infrared stereo camera

DOI: $10.6025 / \mathrm{ed} / 2019 / 8 / 1 / 7-9$

Received: 23 August 2018, Revised 21 October 2018, Accepted 1 November 2018

(C) 2019 DLINE. All Rights Reserved

\section{Introduction}

Parkinson's disease (PD) is a progressive degenerative disease of the extrapyramidal system [1]. The disease often affects people at the age between 35 and 60 years. The following disorders can be observed: rigidity of muscles, slowness of movements (bradykinesia), tremor and various abnormal uncontrolled movements (dyskinesia). Physiotherapy at all stages may help to maintain certain level of quality of life without changing the dosage of medicine. However, only some reports support the statements on successfulness of physiotherapy [2] [3].

In the study we hypothesized that persons with PD can improve their fine motor skills using the 10Cubes3D application regardless of the type of the visual equipment.

\section{Methodology}

Virtual environment (VE) for pinch and grip was created in Unity3D (Unity Technologies, CA, USA). The dedicated space with simulated grass floor was limited with hidden walls and a model of a treasure box. In the middle of the dedicated space 10 cubes with the same physical model, size, virtual weight, bounce stiffness, material, etc., were placed. The goal of the task was to grab

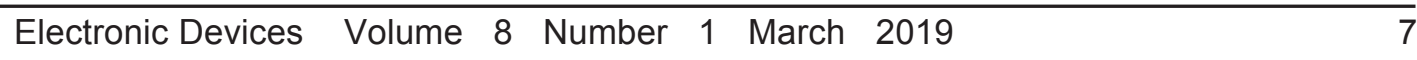


and put all the cubes into the treasure box, oneby- one with the virtual hand. The virtual hand was a VR avatar presentation of the participant's hand that was tracked in realtime by a mini camera (Leap Motion Controller, Leap Motion Inc., CA, USA). The camera tracked hand and fingers position, both required for construction of the 3D VR hand motion (Fig. 1) and the participant can view his/her hand in the VE. We designed an environment for left and right handed participants; the right handed grabbed the cube with the right hand and put it in the box on the left side of the VE and vice-versa for the left handed participants. The software for the assessment and control was written in C\# using Leap Motion libraries. We designed the VE for the use with LCD screen/ laptop and with the 3D goggles (Oculus Rift CV1, Oculus VR, LCC, USA).

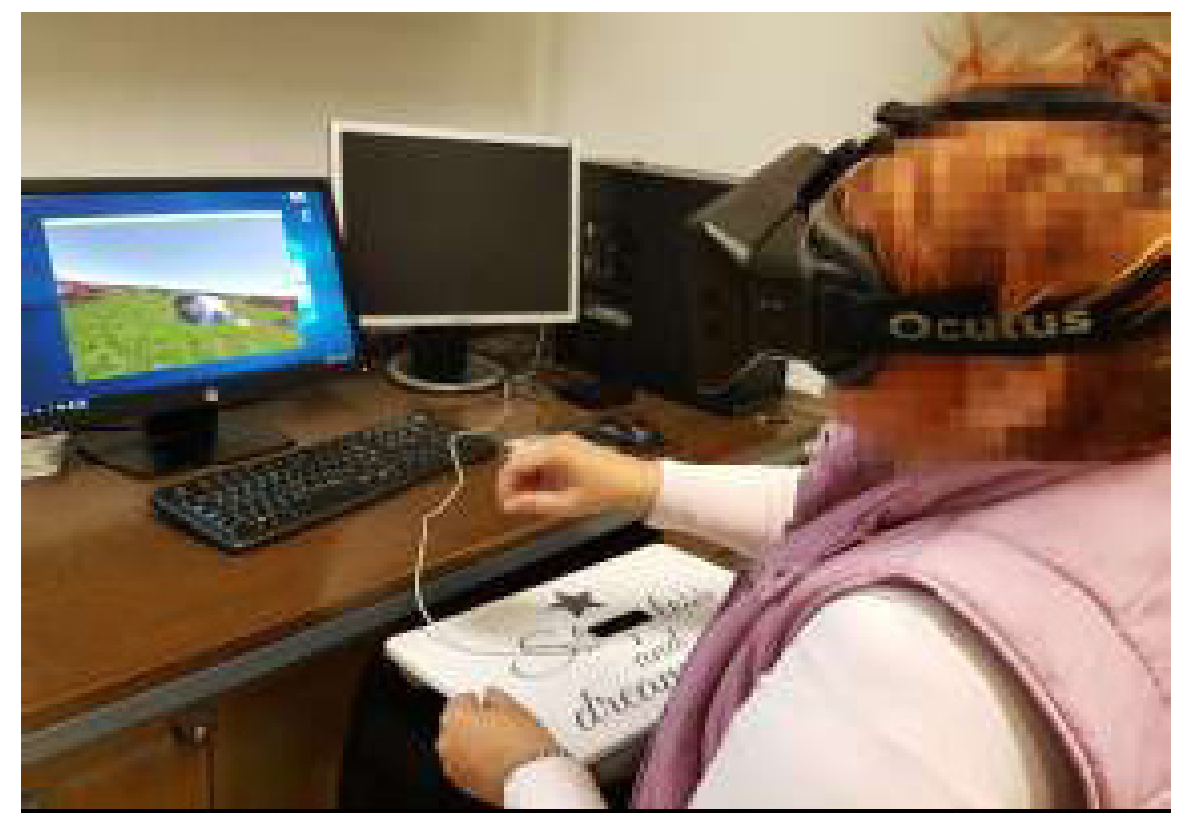

Figure 1. 10Cubes 3D: What are the advantages of the 3D virtual exergaming for persons with PD at home based physiotherapy or telerehabilitation?

In the study 13 persons with Parkinson's disease were involved (67y, 7y after the first symptoms). The participants were randomized into 2 groups; one using the Oculus Rift 3D ( 5 males, 2 females) and the other using a laptop ( 4 males, 2 females). The study was approved by local ethics committee and all participants gave a written consent. We examined the kinematics of the hand movement [4] and the changes in the clinical test Box \& blocks.

\section{Results}

The participants demonstrated higher score at Box \& Blocks clinical test in both groups (4/2, 4/3):

\begin{tabular}{|l|l|l|}
\hline BBT & Affected & Non-affected \\
\hline LCD & $4 / 2 / 0$ & $5 / 1 / 0$ \\
3D & $4 / 3 / 0$ & $4 / 1 / 2$ \\
\hline
\end{tabular}

The kinematic analysis demonstrated that the laptop group gradually performed faster, more efficient (inserted more cubes) with less tremor after the training and the 3D group was indeed more successful in speed performance and efficiency, but with significantly more attempts and higher tremor.

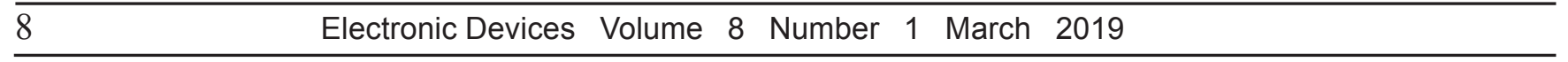




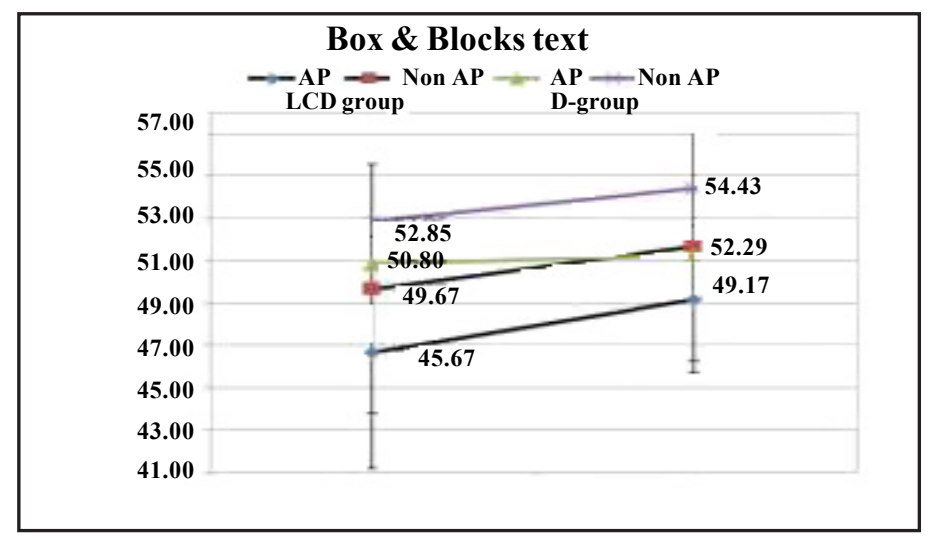

Figure 2. The mean Box \& blocks score improved (more boxes collected, more points) for both groups of participants for affected and non-affected hand

\section{Discussion}

In the preliminary study with only few participants with Parkinson's disease we cannot confirm any major differences between the two applied approaches. Both groups performed well, improved their functional pick and place tasks in the virtual environment and in the real environment as demonstrated by the clinical test. During the task we recorded several unsuccessful trials like misplacement of the cube, cubes falling out of the hand, causing tremendous hand tremor and other measurable components supported by literature [6].

Even if we did not confirm the superiority of the 3D technology over 2D, we would assume that the virtual cube game has enormously increased motivation and thus the participation of the subjects. The motivation of the participant can play an important role [6].

\section{Acknowledgments}

The authors would like to acknowledge the financial support to the Republic of Slovenia and the European Union under the European Regional Development Fund (EkoSMART) and Slovenian Research Agency (project MIO-A J2-7357, program P2-0228).

\section{References}

[1] Melnik M.E. (1995). Basal ganglija disorders. In: Umphred DA ed. Neurological rehabilitation. $3^{\text {rd }}$ ed. St. Louis: Mosby, 606-636.

[2] Clarke CE, Patel S, Ives N, Rick CE, Dowling F, Woolley R, et al. Physiotherapy and Occupational Therapy vs No Therapy in Mild to Moderate Parkinson Disease. JAMA Neurol [Internet]. 2016 Mar;73(3) 291. Available from: http:/www.ncbi.nlm.nih.gov/ pubmed/26785394

[3] Cikajlo, I., Huki, A., Dolinšek I, Zajc D, Vesel M, Krizmani T, et al. Can telerehabilitation games lead to functional improvement of upper extremities in individuals with Parkinson's disease? Int J Rehabil Res [Internet]. 2018 May [cited 2018 Sep 26];41(3)1. Available from: http://www.ncbi.nlm.nih.gov/pubmed/29757774

[4] Barry, G., Galna B., Rochester, L. (2014). The Role of Exergaming in Parkinson's Disease Rehabilitation: A+A Systematic Review of the Evidence. J Neuroeng Rehabil. 7, 11- 33.

[5] Fisher, A.G. 2003. Assessment of Motor and Process Skills: Volume I - Development, Standardization, and Administration Manual Fifth Edition. Three Star Press, Inc.-Fort Collins, Colorado USA

[6] Gorši M, Cikajlo I, Novak D. Competitive and cooperative arm rehabilitation games played by a patient and unimpaired person: Effects on motivation and exercise intensity. J Neuroeng Rehabil [Internet]. 2017 Dec 23 [cited 2017 Apr 11]; 14(1): submitted for publication. Available from: http://www.ncbi.nlm.nih.gov/pubmed/28330504 Figure 2. The mean Box \& blocks score improved (more boxes collected, more points) for both groups of participants for affected and non-affected hand. 